\title{
Asymptotic analysis of thin viscous plate model
}

\author{
I. F. Melikhov, I. Yu. Popov \\ ITMO University, Kronverkskiy, 49, St. Petersburg, 197101, Russia \\ ivan.melikhov@gmail.com; popov1955@gmail.com
}

PACS 47.15.G-,02.30.Jr

DOI 10.17586/2220-8054-2018-9-4-447-456

\begin{abstract}
A cell membrane is a very complex medium, which is difficult to study. One of the simplest approaches is to assume it purely elastic or purely viscous. In this paper, we follow the second assumption and derive mathematical model of nearly-planar viscous plate evolving under action of applied forces. The obtained model is non-linear and covers both stretching and bending of the membrane. In contrast to analogous works on viscous sheets, we use a unique scale for velocity components and take a few first terms in asymptotic expansion. The developed approach can be used for description of the cell membrane with nanoparticles inserted.
\end{abstract}

Keywords: viscous plate, asymptotics.

Received: 15 June 2018

Revised: 5 July 2018

\section{Introduction}

Interest for flows in layers of narrow thickness (nanolayers or microlayers) is due to the rapid development of fluid-based transport processes using in nanotechnologies (see, e.g., [1] and references therein). A special interest in the field is caused by the relation to power systems, medicine, the development of advanced filter elements, etc. For example, the authors of [2] proposed and fabricated a multilayer lateral-flow nanoparticle filtration and separation device. Results of [3] are relevant for water desalination applications. Paper [4] describes a system for ultra-fast mixing of solvents with aqueous fluids and subsequent precipitation of poorly water soluble drug nanoparticles or colloidal carrier particles. Work [5] describes various applications of carbon nanotubes in nanofluidic-based devices. There are great medical challenges in the field. For example, paper [6] studies selective killing of cancer cells by nanoparticle-assisted ultrasound. The author discovered that if the cell membrane contains gold nanoparticles then cancer cell membranes are destroyed by ultrasound with essentially greater probability than that for the corresponding normal cell. To give a theoretical explanation of the phenomenon, one needs a mathematical model. A way to its creation lies through an assumption that the cell membrane is a viscous fluid. Models of such type exist (see, e.g., [7]) but there is no rigorous mathematical background. The aim of this paper is mathematical (more precisely, asymptotic) analysis of viscous plate model. This creates a basis for the following construction of a thin viscous layer containing nanoparticles. One can obtain a solvable model by considering the nanoparticle as a point-like obstacle, so-called stokeslet (see, e.g., [8,9]). The mathematical background of the approach is given by the operator extensions theory (see, e.g., $[10,11]$ ). We will describe the nanoparticle inclusions in the following paper.

Asymptotic analysis is made with respect to small parameter, ratio of the transversal and longitudinal sizes of the viscous layer. Scaling analysis allows us to reveal the orders of terms. We obtained equations describing terms of different orders. Finally, we come back to dimensional form of these equations.

\section{Problem description}

We consider a viscous layer of thickness $h(t, x, y)$, which is bounded by two surfaces $z=H(t, x, y)-$ $h(t, x, y) / 2$ and $z=H(t, x, y)+h(t, x, y) / 2$. Here the center-surface is denoted by $z=H(t, x, y)$. This configuration is illustrated in Fig. 1.

We will study layer's behavior under action of arbitrary volumetric and surface forces. The fluid dynamics is described by Navier-Stokes equations [12]:

$$
\begin{gathered}
\rho\left(\partial_{t} \mathbf{v}+(\mathbf{v} \cdot \nabla) \mathbf{v}\right)=\nabla \cdot \sigma+\mathbf{f}, \\
\nabla \cdot \mathbf{v}=0,
\end{gathered}
$$

where $\rho$ if the fluid density, $\mathbf{v}=(u, v, w)$ is the velocity vector, $\mathbf{f}=\left(f_{x}, f_{y}, f_{z}\right)^{T}$ is the volumetric force vector, $\sigma$ is the stress tensor defined as

$$
\sigma=-p \mathbf{I}+\mu\left(\nabla \mathbf{v}+(\nabla \mathbf{v})^{T}\right)
$$




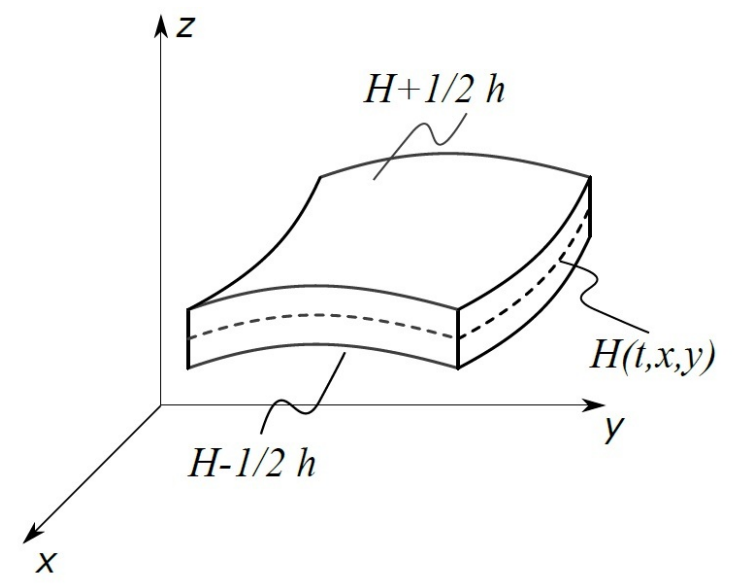

FIG. 1. A viscous layer

where $\mathbf{I}$ is the unit tensor, $p$ is the pressure, $\mu=\mu(x, y)$ is the fluid's dynamic viscosity.

In addition, we set the boundary conditions on the free surfaces. They include the conditions on stress:

$$
\begin{aligned}
& \mathbf{n}^{ \pm} \cdot \sigma^{ \pm} \cdot \mathbf{e}_{x}=F_{x}^{ \pm}, \\
& \mathbf{n}^{ \pm} \cdot \sigma^{ \pm} \cdot \mathbf{e}_{y}=F_{y}^{ \pm}, \\
& \mathbf{n}^{ \pm} \cdot \sigma^{ \pm} \cdot \mathbf{e}_{z}=F_{z}^{ \pm},
\end{aligned}
$$

where $\mathbf{n}^{ \pm}$are normal vectors to the top and bottom surfaces, $\sigma^{ \pm}$are values of the stress tensor on the free surfaces, $\mathbf{e}_{x}, \mathbf{e}_{y}, \mathbf{e}_{z}$ are Cartesian basis vectors, $F_{x}^{ \pm}, F_{y}^{ \pm}, F_{z}^{ \pm}$are applied surface stresses.

Finally, the kinematic conditions take the form:

$$
\partial_{t} H \pm \frac{1}{2} \partial_{t} h+u\left(\partial_{x} H \pm \frac{1}{2} \partial_{x} h\right)+v\left(\partial_{y} H \pm \frac{1}{2} \partial_{y} h\right)-w=0 .
$$

Here the sign "+" is related to the top free surface, and "-" - to the bottom surface.

\section{Scaling analysis}

The governing equations (1)-(7) describes dynamics of the layer, but they are highly nonlinear and difficult to solve. And the main challenge is that due to free surfaces the computation domain is unknown and should be resolved along with velocity and pressure fields. However, we can employ the fact that the considered layer has a small thickness comparing to characteristic length and carry out asymptotic analysis. The first step is scaling analysis.

We define the longitudinal and transversal spatial scales as $L$ and $H_{0}$ correspondingly and introduce parameter $\varepsilon=H_{0} / L$, which we will exploit as a small parameter. As for the velocity scale, we will not vary the longitudinal and transversal directions, as done in the works [13-15]. Instead, we choose a universal scale $U$, which will be discussed later. The viscosity scale is denoted by $\mu_{0}$ and the pressure scale is chosen in the way to meet the scale of viscous stresses.

The dimensionless variables are introduced in the following way:

$$
\begin{array}{rrrrrl}
\tilde{x}=\frac{x}{L} & \tilde{y}=\frac{y}{L}, & \tilde{z}=\frac{z}{H_{0}}, & \tilde{H}=\frac{H}{H_{0}}, & \tilde{h}=\frac{h}{H_{0}}, & \tilde{t}=\frac{H_{0}}{U} t, \\
\tilde{\mu}=\frac{\mu}{\mu_{0}}, & \tilde{u}=\frac{u}{U}, & \tilde{v}=\frac{v}{U}, & \tilde{w}=\frac{w}{U}, & \tilde{p}=\frac{H_{0}}{\mu_{0} U} p, & \\
\tilde{f}_{x}=\frac{f_{x}}{\mathcal{F}_{\|}}, & \tilde{f}_{y}=\frac{f_{y}}{\mathcal{F}_{\|}}, & \tilde{f}_{z}=\frac{f_{z}}{\mathcal{F}_{\perp}}, & \tilde{F}_{x}=\frac{F_{x}}{\mathfrak{F}_{\|}}, & \tilde{F}_{y}=\frac{F_{y}}{\mathfrak{F}_{\|}}, & \tilde{F}_{z}=\frac{F_{z}}{\mathfrak{F}_{\perp},}
\end{array}
$$

where the scales of volumetric forces $\mathcal{F}_{\|}, \mathcal{F}_{\perp}$ and surface stresses $\mathfrak{F}_{\|}, \mathfrak{F}_{\perp}$ will be discussed further. The introduced dimensionless variables imply scales for the stress tensor components. 
The introduced scales imply scaling of the stress tensor $\sigma$ :

$$
\begin{aligned}
& \tilde{\sigma}_{x x}=\frac{H_{0}}{\mu_{0} U} \sigma_{x x}, \quad \tilde{\sigma}_{x y}=\varepsilon \frac{H_{0}}{\mu_{0} U} \sigma_{x y}, \quad \tilde{\sigma}_{y y}=\frac{H_{0}}{\mu_{0} U} \sigma_{y y}, \\
& \tilde{\sigma}_{x z}=\frac{H_{0}}{\mu_{0} U} \sigma_{x z}, \quad \tilde{\sigma}_{y z}=\frac{H_{0}}{\mu_{0} U} \sigma_{y z}, \quad \tilde{\sigma}_{z z}=\frac{H_{0}}{\mu_{0} U} \sigma_{z z} \text {. }
\end{aligned}
$$

Further we will omit the sign ${ }^{\circ}$ over dimensionless variables in the sake of simplicity.

Components of the stress tensor are expressed as:

$$
\begin{array}{lll}
\sigma_{x x}=-p+2 \varepsilon \mu \partial_{x} u, & \sigma_{y y}=-p+2 \varepsilon \mu \partial_{y} v, & \sigma_{z z}=-p+2 \mu \partial_{z} w, \\
\sigma_{x y}=\sigma_{y x}=\mu\left(\partial_{y} u+\partial_{x} v\right), & \sigma_{x z}=\sigma_{z x}=\mu\left(\partial_{z} u+\varepsilon \partial_{x} w\right), & \sigma_{y z}=\sigma_{z y}=\mu\left(\partial_{z} v+\varepsilon \partial_{y} w\right) .
\end{array}
$$

The equations (1)-(2) takes the dimensional form:

$$
\begin{gathered}
R e I_{u}=\varepsilon \partial_{x} \sigma_{x x}+\varepsilon^{2} \partial_{y} \sigma_{x y}+\partial_{z} \sigma_{x z}+\frac{H_{0}^{2} \mathcal{F}_{\|}}{\mu_{0} U} f_{x}, \\
R e I_{v}=\varepsilon^{2} \partial_{x} \sigma_{y x}+\varepsilon \partial_{y} \sigma_{y y}+\partial_{z} \sigma_{y z}+\frac{H_{0}^{2} \mathcal{F}_{\|}}{\mu_{0} U} f_{y}, \\
\operatorname{Re} I_{w}=\varepsilon \partial_{x} \sigma_{z x}+\varepsilon \partial_{y} \sigma_{z y}+\partial_{z} \sigma_{z z}+\frac{H_{0}^{2} \mathcal{F}_{\perp}}{\mu_{0} U} f_{z}, \\
\varepsilon \partial_{x} u+\varepsilon \partial_{y} v+\partial_{z} w=0
\end{gathered}
$$

where Reynold number Re is defined as:

$$
R e=\frac{\rho U H_{0}}{\mu_{0}}
$$

and the inertial terms are denoted as:

$$
\begin{gathered}
I_{u}=\left(\partial_{t} u+\varepsilon u \partial_{x} u+\varepsilon v \partial_{y} u+w \partial_{z} u\right), \\
I_{v}=\left(\partial_{t} v+\varepsilon u \partial_{x} v+\varepsilon v \partial_{y} v+w \partial_{z} v\right), \\
I_{w}=\left(\partial_{t} w+\varepsilon u \partial_{x} w+\varepsilon v \partial_{y} w+w \partial_{z} w\right) .
\end{gathered}
$$

The boundary conditions (4)-(6) takes the form:

$$
\begin{gathered}
\sigma_{x z}-\varepsilon \sigma_{x x}\left(\partial_{x} H \pm \frac{1}{2} \partial_{x} h\right)-\varepsilon^{2} \sigma_{x y}\left(\partial_{y} H \pm \frac{1}{2} \partial_{y} h\right)=\mp \frac{H_{0} \mathfrak{F}_{\|}}{\mu_{0} U} \frac{F_{x}^{ \pm}}{N^{ \pm}} \\
\sigma_{y z}-\varepsilon^{2} \sigma_{y x}\left(\partial_{x} H \pm \frac{1}{2} \partial_{x} h\right)-\varepsilon \sigma_{y y}\left(\partial_{y} H \pm \frac{1}{2} \partial_{y} h\right)=\mp \frac{H_{0} \mathfrak{F}_{\|}}{\mu_{0} U} \frac{F_{y}^{ \pm}}{N^{ \pm}} \\
\sigma_{z z}-\varepsilon \sigma_{z x}\left(\partial_{x} H \pm \frac{1}{2} \partial_{x} h\right)-\varepsilon \sigma_{z y}\left(\partial_{y} H \pm \frac{1}{2} \partial_{y} h\right)=\mp \frac{H_{0} \mathfrak{F}_{\perp}}{\mu_{0} U} \frac{F_{z}^{ \pm}}{N^{ \pm}}
\end{gathered}
$$

where:

$$
N^{ \pm}=\left(1+\varepsilon^{2}\left(\partial_{x} H+\frac{1}{2} \partial_{x} h\right)^{2}+\varepsilon^{2}\left(\partial_{y} H+\frac{1}{2} \partial_{y} h\right)^{2}\right)^{-1 / 2} .
$$

Kinematic condition (7) is rewritten as:

$$
w=\partial_{t} H \pm \frac{1}{2} \partial_{t} h+\varepsilon\left[u\left(\partial_{x} H \pm \frac{1}{2} \partial_{x} h\right)+v\left(\partial_{y} H \pm \frac{1}{2} \partial_{y} h\right)\right]
$$

\section{Viscous plate equations}

The equation (9) can be treated as a first order equation on $\sigma_{x z}$, which can be solved, giving:

$$
\sigma_{x z}=\left.\sigma_{x z}\right|_{-}+\int^{z}\left[R e I_{u}-\varepsilon \partial_{x} \sigma_{x x}-\varepsilon^{2} \partial_{y} \sigma_{x y}-\frac{H_{0}^{2} \mathcal{F}_{\|}}{\mu_{0} U} f_{x}\right] d z^{\prime},
$$


where $\left.\sigma_{x z}\right|_{-}$is the value of $\sigma_{x z}$ at the bottom surface $H-h / 2$ taken from the boundary conditions, and the lower integration limit $i i-i j$ is denoted to the surface $H-h / 2$. In addition, this solution should satisfy the boundary conditions on the top surface $H+h / 2$. It leads to the solvability condition of the equation (9):

$$
\left.\sigma_{x z}\right|_{+}=\left.\sigma_{x z}\right|_{-}+\int_{-}^{+}\left[R e I_{u}-\varepsilon \partial_{x} \sigma_{x x}-\varepsilon^{2} \partial_{y} \sigma_{x y}-\frac{H_{0}^{2} \mathcal{F}_{\|}}{\mu_{0} U} f_{x}\right] d z,
$$

where the integrals are taken over the interval from $H-h / 2$ to $H+h / 2$.

Using the rule of integral differentiation and accounting for boundary conditions (16), we come to the following form of the solvability condition:

$$
\varepsilon \partial_{x}\left[\int_{-}^{+} \sigma_{x x} d z\right]+\varepsilon^{2} \partial_{y}\left[\int_{-}^{+} \sigma_{x y} d z\right]=R e \bar{I}_{u}-\frac{H_{0}^{2} \mathcal{F}_{\|}}{\mu_{0} U} \bar{f}_{x}+\frac{H_{0} \mathfrak{F}_{\|}}{\mu_{0} U}\left(\frac{F_{x}^{+}}{N^{+}}+\frac{F_{x}^{-}}{N^{-}}\right),
$$

where $\bar{I}_{u}, \bar{f}_{x}$ are thickness-averaged inertia terms and volumetric force:

$$
\bar{I}_{u}=\int_{-}^{+} I_{u} d z, \quad \bar{f}_{x}=\int_{-}^{+} f_{x} d z
$$

Treating two other components of equation (9) in the same way we get the expressions of $\sigma_{y z}, \sigma_{z z}$ :

$$
\begin{gathered}
\sigma_{y z}=\left.\sigma_{y z}\right|_{-}+\int_{-}^{z}\left[R e I_{v}-\varepsilon^{2} \partial_{x} \sigma_{y x}-\varepsilon \partial_{y} \sigma_{y y}-\frac{H_{0}^{2} \mathcal{F}_{\|}}{\mu_{0} U} f_{y}\right] d z^{\prime} \\
\sigma_{z z}=\left.\sigma_{z z}\right|_{-}+\int_{-}^{z}\left[R e I_{w}-\varepsilon \partial_{x} \sigma_{z x}-\varepsilon \partial_{y} \sigma_{z y}-\frac{H_{0}^{2} \mathcal{F}_{\perp}}{\mu_{0} U} f_{z}\right] d z^{\prime}
\end{gathered}
$$

and solviability conditions for (10)-(11):

$$
\begin{aligned}
& \varepsilon^{2} \partial_{x}\left[\int_{-}^{+} \sigma_{y x} d z\right]+\varepsilon \partial_{y}\left[\int_{-}^{+} \sigma_{y y} d z\right]=\operatorname{Re} \bar{I}_{v}-\frac{H_{0}^{2} \mathcal{F}_{\|}}{\mu_{0} U} \bar{f}_{y}+\frac{H_{0} \mathfrak{F}_{\|}}{\mu_{0} U}\left(\frac{F_{y}^{+}}{N^{+}}+\frac{F_{y}^{-}}{N^{-}}\right) \\
& \varepsilon \partial_{x}\left[\int_{-}^{+} \sigma_{z x} d z\right]+\varepsilon \partial_{y}\left[\int_{-}^{+} \sigma_{z y} d z\right]=\operatorname{Re} \bar{I}_{w}-\frac{H_{0}^{2} \mathcal{F}_{\perp}}{\mu_{0} U} \bar{f}_{z}+\frac{H_{0} \mathfrak{F}_{\perp}}{\mu_{0} U}\left(\frac{F_{z}^{+}}{N^{+}}+\frac{F_{z}^{-}}{N^{-}}\right) .
\end{aligned}
$$

Consider the integral $\int_{-}^{+} \sigma_{z x} d z$, which after differentiation by parts and accounting for (9) can be written as:

$$
\begin{aligned}
\int_{-}^{+} \sigma_{z x} d z=\left.(z-H) \sigma_{z x}\right|_{-} ^{+}-\int_{-}^{+}(z-H) \partial_{z} \sigma_{z x} d z= & \\
=\left.(z-H) \sigma_{z x}\right|_{-} ^{+}+\varepsilon \int_{-}^{+}(z-H) \partial_{x} \sigma_{x x} d z+\varepsilon^{2} \int_{-}^{+}(z-H) \partial_{y} \sigma_{x y} d z+ & \\
& +\frac{H_{0}^{2} \mathcal{F}_{\|}}{\mu_{0} U} \int_{-}^{+}(z-H) f_{x} d z-\operatorname{Re} \int_{-}^{+}(z-H) I_{u} d z .
\end{aligned}
$$


Applying integral differentiation rule we get:

$$
\begin{aligned}
& \int_{-}^{+} \sigma_{z x} d z= \\
& \quad=\varepsilon \partial_{x}\left[\int_{-}^{+}(z-H) \sigma_{x x} d z\right]+\varepsilon^{2} \partial_{y}\left[\int_{-}^{+}(z-H) \sigma_{x y} d z\right]_{-}^{+}+\partial_{x} H \int_{-}^{+} \sigma_{x x} d z+\varepsilon^{2} \partial_{y} H \int_{-}^{+} \sigma_{x y} d z+ \\
& \left.\quad+\frac{H_{0}^{2} \mathcal{F}_{\|}}{\mu_{0} U} \int_{-}^{+}(z-H) f_{x} d z-R e I_{-}^{+}(z-H) I_{u} d z-\frac{H_{0} \mathfrak{F}_{\|}}{\mu_{0} U} \frac{F_{x}^{+}}{N^{+}}-\frac{F_{x}^{-}}{N^{-}}\right)
\end{aligned}
$$

Calculating $\int_{-}^{+} \sigma_{z y} d z$ in the same way and substituting the obtained expressions in (26), we get the final form of the third solvability condition:

$$
\begin{aligned}
& \varepsilon \partial_{x}\left\{\varepsilon \partial_{x}\left[\int_{-}^{+}(z-H) \sigma_{x x} d z\right]+\varepsilon^{2} \partial_{y}\left[\int_{-}^{+}(z-H) \sigma_{x y} d z\right]+\right. \\
& \left.+\varepsilon \partial_{x} H \int_{-}^{+} \sigma_{x x} d z+\varepsilon^{2} \partial_{y} H \int_{-}^{+} \sigma_{x y} d z\right\}+ \\
& +\varepsilon \partial_{y}\left\{\varepsilon^{2} \partial_{x}\left[\int_{-}^{+}(z-H) \sigma_{y x} d z\right]+\varepsilon \partial_{y}\left[\int_{-}^{+}(z-H) \sigma_{y y} d z\right]+\right. \\
& \left.+\varepsilon^{2} \partial_{x} H \int_{-}^{+} \sigma_{y x} d z+\varepsilon \partial_{y} H \int_{-}^{+} \sigma_{y y} d z\right\}= \\
& =-\frac{H_{0}^{2} \mathcal{F}_{\perp}}{\mu_{0} U} \bar{f}_{z}-\varepsilon \frac{H_{0}^{2} \mathcal{F}_{\|}}{\mu_{0} U}\left[\partial_{x}\left(\int_{-}^{+}(z-H) f_{x} d z\right)+\partial_{y}\left(\int_{-}^{+}(z-H) f_{y} d z\right)\right]+ \\
& +\frac{H_{0} \mathfrak{F}_{\perp}}{\mu_{0} U}\left(\frac{F_{z}^{+}}{N^{+}}+\frac{F_{z}^{-}}{N^{-}}\right)+\varepsilon \frac{H_{0} \mathfrak{F}_{\|}}{\mu_{0} U}\left[\partial_{x}\left(\frac{h}{2}\left(\frac{F_{x}^{+}}{N^{+}}-\frac{F_{x}^{-}}{N^{-}}\right)\right)+\partial_{y}\left(\frac{h}{2}\left(\frac{F_{y}^{+}}{N^{+}}-\frac{F_{y}^{-}}{N^{-}}\right)\right)\right]+ \\
& +\operatorname{Re}\left\{\bar{I}_{w}+\varepsilon \partial_{x}\left[\int_{-}^{+}(z-H) I_{u} d z\right]+\varepsilon \partial_{y}\left[\int_{-}^{+}(z-H) I_{v} d z\right]\right\} .
\end{aligned}
$$

Further, we will consider systems with $R e=O\left(\varepsilon^{4}\right)$. The force scales $\mathcal{F}_{\|}, \mathcal{F}_{\perp}, \mathfrak{F}_{\|}, \mathfrak{F}_{\perp}$ we define from (22), (25), (28). We focus on the cases, when external forces are balanced by viscous stresses, which implies:

$$
\begin{array}{rlrl}
\mathcal{F}_{\|} & =\varepsilon^{2} \frac{\mu_{0} U}{H_{0}^{2}}, & \mathfrak{F}_{\|} & =\varepsilon^{2} \frac{\mu_{0} U}{H_{0}}, \\
\mathcal{F}_{\perp} & =\varepsilon^{3} \frac{\mu_{0} U}{H_{0}^{2}}, & \mathfrak{F}_{\perp}=\varepsilon^{3} \frac{\mu_{0} U}{H_{0}} .
\end{array}
$$

In addition to the equations of dynamics (9)-(11), we consider continuity equation (12). Integrating it with respect to $z$-coordinate, we find:

$$
w=\left.w\right|_{-}-\varepsilon \int_{-}^{z} \partial_{x} u+\partial_{y} v d z .
$$

As before, applying boundary conditions on the top surface leads to the solvability condition:

$$
\partial_{t} h+\varepsilon \partial_{x}(h \bar{u})+\varepsilon \partial_{y}(h \bar{v})=0,
$$


where thickness-averaged velocities $\bar{u}, \bar{v}$ are found as:

$$
\bar{u}=\frac{1}{h} \int_{-}^{+} u d z, \quad \bar{v}=\frac{1}{h} \int_{-}^{+} v d z .
$$

Finally, we find the form of average vertical velocity $\bar{w}$. Integrating the expression (29), employing the equation (12) and using the boundary conditions we get:

$$
\bar{w}=\partial_{t} H+\varepsilon h^{-1}\left[\partial_{x}\left(\int_{-}^{+}(z-H) u d z\right)+\partial_{x} H \int_{-}^{+} u d z+\partial_{y}\left(\int_{-}^{+}(z-H) v d z\right)+\partial_{y} H \int_{-}^{+} v d z\right] .
$$

Note that, in general, the average velocity $\bar{w}$ is not equal to the velocity of the center surface $\partial_{t} H$.

In the subsequent analysis, we will focus on the cases, when $F_{x}^{ \pm}=F_{y}^{ \pm}=0 \quad \partial_{z} f_{x}=\partial_{z} f_{y}=0$. Then the equations (22), (25), (28) can be written in simpler form, similar to elastic plate equations:

$$
\begin{gathered}
\varepsilon^{-1} \partial_{x} N_{x x}+\partial_{y} N_{x y}=\varepsilon^{2} R e^{*} \bar{I}_{u}-\bar{f}_{x} \\
\partial_{x} N_{y x}+\varepsilon^{-1} \partial_{y} N_{y y}=\varepsilon^{2} R e^{*} \bar{I}_{v}-\bar{f}_{y}, \\
\partial_{x} Q_{x}+\partial_{y} Q_{y}=\varepsilon R e^{*}\left[\bar{I}_{w}+\varepsilon\left(\partial_{x} \overline{\bar{I}}_{u}+\partial_{y} \overline{\bar{I}}_{v}\right)\right]-\bar{f}_{z}+\left(\frac{F_{z}^{+}}{N^{+}}+\frac{F_{z}^{-}}{N^{-}}\right),
\end{gathered}
$$

where the effective in-plane stresses are defined as:

$$
N_{x x}=\int_{-}^{+} \sigma_{x x} d z, \quad N_{y y}=\int_{-}^{+} \sigma_{y y} d z, \quad N_{x y}=N_{y x}=\int_{-}^{+} \sigma_{x y} d z,
$$

the residual forces are:

$$
\begin{aligned}
& Q_{x}=\varepsilon^{-1} \partial_{x} M_{x x}+\varepsilon^{-1} N_{x x} \partial_{x} H+\partial_{y} M_{x y}+N_{x y} \partial_{y} H \\
& Q_{y}=\partial_{x} M_{y x}+N_{y x} \partial_{x} H+\varepsilon^{-1} \partial_{y} M_{y y}+\varepsilon^{-1} N_{y y} \partial_{y} H
\end{aligned}
$$

and bending moments are:

$$
\begin{gathered}
M_{x x}=\int_{-}^{+}(z-H) \sigma_{x x} d z, \\
M_{x y}=M_{y x}=\int_{-}^{+}(z-H) \sigma_{x y} d z, \\
M_{y y}=\int_{-}^{+}(z-H) \sigma_{y y} d z,
\end{gathered}
$$

symbols $\overline{\bar{I}}_{u}, \overline{\bar{I}}_{v}, \overline{\bar{I}}_{w}$ are denoted to the moments of inertial forces:

$$
\overline{\bar{I}}_{u}=\int_{-}^{+}(z-H) I_{u} d z, \quad \overline{\bar{I}}_{v}=\int_{-}^{+}(z-H) I_{v} d z, \quad \overline{\bar{I}}_{w}=\int_{-}^{+}(z-H) I_{w} d z
$$

\section{Asymptotic solution}

Up to know we just rearranged non-dimensional Navier-Stokes equations without using the small parameter expansion. Now we are going to find velocity distribution through thickness, expanding the variables on small parameter:

$$
A=A^{(0)}+\varepsilon A^{(1)}+\varepsilon^{2} A^{(2)}+o\left(\varepsilon^{2}\right) .
$$

From the equations (21), (23) we have in zero order

$$
\begin{aligned}
& \sigma_{x z}^{(0)}=0, \\
& \sigma_{y z}^{(0)}=0,
\end{aligned}
$$


or

$$
\begin{aligned}
& \mu \partial_{z} u^{(0)}=0, \\
& \mu \partial_{z} v^{(0)}=0
\end{aligned}
$$

which has the following solution:

$$
\begin{aligned}
& u^{(0)}=\bar{u}^{(0)}(t, x, y), \\
& v^{(0)}=\bar{v}^{(0)}(t, x, y) .
\end{aligned}
$$

The continuity equation (12) implies:

$$
\partial_{z} w^{(0)}=0
$$

thus

$$
w^{(0)}=\bar{w}^{(0)}(t, x, y)
$$

Finally, for $z z$-stress in zero order, we have:

$$
\sigma_{z z}^{(0)}=0
$$

therefore

or, taking into account (46),

$$
-p^{(0)}+2 \mu \partial_{z} w^{(0)}=0
$$

$$
p^{(0)}=0
$$

Note that:

$$
\sigma_{x x}^{(0)}=\sigma_{y y}^{(0)}=-p^{(0)}=0 .
$$

Since the equations (32)-(34) include terms of the order $\varepsilon^{-1}$, the obtained expressions are not enough. We have to continue with analysis of the first order.

Due to (21) the stress $\sigma_{x z}$ in the first order is equal to

$$
\sigma_{x z}^{(1)}=\left.\sigma_{x x}^{(0)}\right|_{-}\left(\partial_{x} H-\frac{1}{2} \partial_{x} h\right)-\int_{-}^{+} \partial_{x} \sigma_{x x}^{(0)} d z,
$$

where the right-hand side is zero. Thus:

$$
\mu\left(\partial_{z} u^{(1)}+\partial_{x} \bar{w}^{(0)}\right)=0
$$

And we get that:

$$
u^{(1)}=\partial_{x} \bar{w}^{(0)}(H-z)+\bar{u}^{(1)} .
$$

In the same way, we obtain that:

$$
\begin{gathered}
\sigma_{x z}^{(1)}=0, \\
v^{(1)}=\partial_{y} \bar{w}^{(0)}(H-z)+\bar{v}^{(1)} .
\end{gathered}
$$

The continuity equation (12) in the first order is written as:

$$
\partial_{z} w^{(1)}=-\partial_{x} u^{(0)}-\partial_{y} v^{(0)} .
$$

The value of $\partial_{z} w^{(1)}$ will be used for calculating the stress $\sigma_{z z}^{(1)}$, which is equal to:

$$
\sigma_{z z}^{(1)}=\left.\sigma_{z x}^{(0)}\right|_{-}\left(\partial_{x} H-\frac{1}{2} \partial_{x} h\right)+\left.\sigma_{z y}^{(0)}\right|_{-}\left(\partial_{y} H-\frac{1}{2} \partial_{y} h\right)-\int_{-}^{+}\left(\partial_{x} \sigma_{z x}^{(0)}+\partial_{y} \sigma_{z y}^{(0)}\right) d z
$$

where the right-hand side vanishes. Therefore:

$$
-p^{(1)}+2 \mu \partial_{z} w^{(1)}=0
$$

and taking into account (53) we get

$$
p^{(1)}=-2 \mu\left(\partial_{x} u^{(0)}+\partial_{y} v^{(0)}\right) .
$$

As for the next order, $\varepsilon^{2}$, we consider only the continuity equation:

$$
\partial_{z} w^{(2)}=-\partial_{x} u^{(1)}-\partial_{y} v^{(1)}
$$


and equation (24):

$$
\sigma_{z z}^{(2)}=\left.\sigma_{z x}^{(1)}\right|_{-}\left(\partial_{x} H-\frac{1}{2} \partial_{x} h\right)+\left.\sigma_{z y}^{(1)}\right|_{-}\left(\partial_{y} H-\frac{1}{2} \partial_{y} h\right)-\int_{-}^{+}\left(\partial_{x} \sigma_{z x}^{(1)}+\partial_{y} \sigma_{z y}^{(1)}\right) d z
$$

where the right-hand side is zero again. Therefore, we get:

$$
p^{(2)}=-2 \mu\left(\partial_{x} u^{(1)}+\partial_{y} v^{(1)}\right) .
$$

Finally, using the expression for longitudinal velocity $u$ (44), (50) and $v$ (45), (52), pressure $p$ (48), (55), (58) we obtain stress distributions across thickness

$$
\begin{aligned}
\begin{aligned}
\sigma_{x x}=2 \varepsilon \mu\left\{2 \partial _ { x } \left[\bar{u}^{(0)}+\varepsilon\left(\partial_{x}\left(\bar{w}^{(0)}(H-z)\right)+\right.\right.\right. & \left.\left.\bar{u}^{(1)}\right)\right]+ \\
& \left.+\partial_{y}\left[\bar{v}^{(0)}+\varepsilon\left(\partial_{y}\left(\bar{w}^{(0)}(H-z)\right)+\bar{v}^{(1)}\right)\right]\right\}+O\left(\varepsilon^{3}\right),
\end{aligned} \\
\begin{aligned}
\sigma_{x y}=\mu\left\{\partial_{y}\left[\bar{u}^{(0)}+\varepsilon\left(\partial_{x}\left(\bar{w}^{(0)}(H-z)\right)+\bar{u}^{(1)}\right)\right]\right. & + \\
& \left.+\partial_{x}\left[\bar{v}^{(0)}+\varepsilon\left(\partial_{y}\left(\bar{w}^{(0)}(H-z)\right)+\bar{v}^{(1)}\right)\right]\right\}+O\left(\varepsilon^{2}\right), \\
\sigma_{y y}=2 \varepsilon \mu\left\{\partial _ { x } \left[\bar{u}^{(0)}+\varepsilon\left(\partial_{x}\left(\bar{w}^{(0)}(H-z)\right)+\right.\right.\right. & \left.\left.\bar{u}^{(1)}\right)\right]+ \\
& \left.+2 \partial_{y}\left[\bar{v}^{(0)}+\varepsilon\left(\partial_{y}\left(\bar{w}^{(0)}(H-z)\right)+\bar{v}^{(1)}\right)\right]\right\}+O\left(\varepsilon^{3}\right) .
\end{aligned}
\end{aligned}
$$

Integrating it through thickness, we find effective stresses:

$$
\begin{gathered}
N_{x x}=2 \varepsilon \mu h\left\{2\left[\partial_{x}\left(\bar{u}^{(0)}+\varepsilon \bar{u}^{(1)}\right)+\varepsilon \partial_{x} \bar{w}^{(0)} \partial_{x} H\right]+\left[\partial_{y}\left(\bar{v}^{(0)}+\varepsilon \bar{v}^{(1)}\right)+\varepsilon \partial_{y} \bar{w}^{(0)} \partial_{y} H\right]\right\}+O\left(\varepsilon^{3}\right), \\
N_{x y}=\mu h\left[\partial_{y}\left(\bar{u}^{(0)}+\varepsilon \bar{u}^{(1)}\right)+\partial_{x}\left(\bar{v}^{(0)}+\varepsilon \bar{v}^{(1)}\right)+\varepsilon\left(\partial_{x} \bar{w}^{(0)} \partial_{y} H+\partial_{y} \bar{w}^{(0)} \partial_{x} H\right)\right]+O\left(\varepsilon^{2}\right), \\
N_{y y}=2 \varepsilon \mu h\left\{\left[\partial_{x}\left(\bar{u}^{(0)}+\varepsilon \bar{u}^{(1)}\right)+\varepsilon \partial_{x} \bar{w}^{(0)} \partial_{x} H\right]+2\left[\partial_{y}\left(\bar{v}^{(0)}+\varepsilon \bar{v}^{(1)}\right)+\varepsilon \partial_{y} \bar{w}^{(0)} \partial_{y} H\right]\right\}+O\left(\varepsilon^{3}\right) .
\end{gathered}
$$

and bending moments:

$$
\begin{gathered}
M_{x x}=-\varepsilon^{2} \frac{\mu h^{3}}{6}\left(2 \partial_{x x}^{2} \bar{w}^{(0)}+\partial_{y y}^{2} \bar{w}^{(0)}\right)+O\left(\varepsilon^{3}\right), \\
M_{x y}=-\varepsilon \frac{\mu h^{3}}{6} \partial_{x y}^{2} \bar{w}^{(0)}+O\left(\varepsilon^{2}\right), \\
M_{y y}=-\varepsilon^{2} \frac{\mu h^{3}}{6}\left(\partial_{x x}^{2} \bar{w}^{(0)}+2 \partial_{y y}^{2} \bar{w}^{(0)}\right)+O\left(\varepsilon^{3}\right) .
\end{gathered}
$$

Substituting (62)-(67) in equations (32)-(34), we obtain with accuracy of $O\left(\varepsilon^{2}\right)$

$$
\begin{gathered}
\varepsilon^{-1} \partial_{x} N_{x x}+\partial_{y} N_{x y}=-\bar{f}_{x}, \\
\partial_{x} N_{y x}+\varepsilon^{-1} \partial_{y} N_{y y}=-\bar{f}_{y}, \\
\partial_{x} Q_{x}+\partial_{y} Q_{y}=\varepsilon R e^{*} \partial_{t} w^{(0)}-\bar{f}_{z}+\left(F_{z}^{+}+F_{z}^{-}\right) .
\end{gathered}
$$

Then, the continuity equation (30) takes the form:

$$
\partial_{t} h+\varepsilon \partial_{x}\left(h \bar{u}^{(0)}\right)+\varepsilon \partial_{y}\left(h \bar{v}^{(0)}\right)=0,
$$

and the average vertical velocity is equal to the center surface velocity:

$$
\bar{w}^{(0)}=\partial_{t} H .
$$




\section{Dimensional equations}

In this section, we come back to dimensional variables. First, we collect the expansion for average velocities:

$$
\begin{gathered}
\bar{u}=\bar{u}^{(0)}+\varepsilon \bar{u}^{(1)}+O\left(\varepsilon^{2}\right), \\
\bar{v}=\bar{v}^{(0)}+\varepsilon \bar{v}^{(1)}+O\left(\varepsilon^{2}\right), \\
\bar{w}=\bar{w}^{(0)}+O(\varepsilon) .
\end{gathered}
$$

Then we can rearrange the equation and rewrite them in dimensional form:

$$
\begin{gathered}
\partial_{t} h+\partial_{x}(h \bar{u})+\partial_{y}(h \bar{v})=0, \\
\partial_{x} N_{x x}+\partial_{y} N_{x y}=-\bar{f}_{x}, \\
\partial_{x} N_{y x}+\partial_{y} N_{y y}=-\bar{f}_{y}, \\
\partial_{x} Q_{x}+\partial_{y} Q_{y}=\rho h \partial_{t} \bar{w}-\bar{f}_{z}+\left(F_{z}^{+}+F_{z}^{-}\right),
\end{gathered}
$$

where the membrane stresses are defined as:

$$
\begin{gathered}
N_{x x}=2 \mu h\left[2\left(\partial_{x} \bar{u}+\partial_{x} \bar{w} \partial_{x} H\right)+\left(\partial_{y} \bar{v}+\partial_{y} \bar{w} \partial_{y} H\right)\right], \\
N_{x y}=\mu h\left(\partial_{y} \bar{u}+\partial_{x} \bar{w} \partial_{y} H+\partial_{x} \bar{v}+\partial_{y} \bar{w} \partial_{x} H\right), \\
N_{y y}=2 \mu h\left[\left(\partial_{x} \bar{u}+\partial_{x} \bar{w} \partial_{x} H\right)+2\left(\partial_{y} \bar{v}+\partial_{y} \bar{w} \partial_{y} H\right)\right] .
\end{gathered}
$$

the residual forces depend on bending moments and membrane stresses:

$$
\begin{aligned}
& Q_{x}=\partial_{x} M_{x x}+\partial_{y} M_{x y}+N_{x x} \partial_{x} H+N_{x y} \partial_{y} H, \\
& Q_{y}=\partial_{x} M_{y x}+\partial_{y} M_{y y}+N_{y x} \partial_{x} H+N_{y y} \partial_{y} H,
\end{aligned}
$$

and the bending moments are calculated as:

$$
\begin{gathered}
M_{x x}=-\frac{\mu h^{3}}{6}\left(2 \partial_{x x}^{2} \bar{w}+\partial_{y y}^{2} \bar{w}\right), \\
M_{x y}=-\frac{\mu h^{3}}{6} \partial_{x y}^{2} \bar{w}, \\
M_{y y}=-\frac{\mu h^{3}}{6}\left(\partial_{x x}^{2} \bar{w}+2 \partial_{y y}^{2} \bar{w}\right) .
\end{gathered}
$$

The average vertical velocity is expressed as:

$$
\bar{w}=\partial_{t} H .
$$

Finally, the average volumetric forces are calculated in the following way:

$$
\bar{f}_{x}=\int_{-}^{+} f_{x} d z, \quad \bar{f}_{y}=\int_{-}^{+} f_{y} d z, \quad \bar{f}_{z}=\int_{-}^{+} f_{z} d z .
$$

Equations (73)-(86) form the viscous plate model. They include terms up to the order of $O(\varepsilon)$. The variables to solve for are the averaged velocities $\bar{u}, \bar{v}$, the deflection $H$ and the thickness $h$. However, from computational point of view it is convenient to keep the unknown variable $\bar{w}$.

For the finite plate, the equations should be accompanied by boundary conditions along the plate's perimeter. It can be clamped, hinged or free conditions similar to those for elastic plates.

\section{Discussion}

During the model derivation we made the assumption that both deflection $H$ and thickness $h$ are of the same scale $H_{0}$, which is much smaller than plate's length. However, we didn't impose any restrictions on the ratio $H / h$. In contrast, classical elastic plate linear theory is applicable only in the cases $H / h \ll 1$. Indeed, the derived equations are analogous to non-linear elastic plate theory (see, e.g., [16]). It is worth noting that inertial effects appears even for quite small Reynolds numbers $R e \sim \varepsilon^{4}$.

As we mentioned before, there are two classical problems regarding viscous plate: stretching and bending. These two effects are described by different scales for velocities. In order to cover them within one model, these problems were solved separately and then matching asymptotic series was done [13-15]. Our approach has the benefit of the unique velocity scale.

Let's consider the system with no transversal forces $f_{z}, F_{z}^{ \pm}$. In this case, it is enough to consider only $\bar{u}^{(0)}$, $\bar{v}^{(0)}$ for calculation of (59)-(61). Then non-linear terms in (62)-(64), which include $\bar{w}^{(0)}$, are of higher order 
and can be neglected. The bending moments (65)-(67) vanishes, and equation (70) implies $H=0$. Thus, plate's stretching is described by continuity equation (73) and stress balance equations (74)-(75).

Pure bending can be considered when applied longitudinal forces are zero. Then zero-order equations (32)-(32) have the only solution $\bar{u}^{(0)}=\bar{v}^{(0)}=0$, which together with (71) imply constant-in-time thickness $\partial_{t} h=0$. The corresponding longitudinal velocities becomes of order $\varepsilon$.

In more involved cases bending and stretching occurs simultaneously. In particular, it happens in the cases of large deflections, when bending implies stretching. The latter effectively makes the system stiffer.

Different external influences can be treated as applied forces. For example, nanoparticles can be seen as pointwise forces, applied at the surface.

\section{Acknowledgements}

This work was partially financially supported by the Government of the Russian Federation (grant 08-08), by grant 16-11-10330 of Russian Science Foundation.

\section{References}

[1] Rudyak V., Belkin A. Molecular dynamics simulation of fluid viscosity in nanochannels. Nanosystems: Phys. Chem. Math., 2018, 9(3), P. 349-355.

[2] Jiang Y., Yu Z., Huang X., Chen R., Chen W., Zeng Y., Xu Ch., Min H., Zheng N., Cheng X. A multilayer lateral-flow microfluidic device for particle separation. Microfluid Nanofluid, 2018, 22, P. 40.

[3] Celebi A.T., Barisik M., Beskok A. Surface charge-dependent transport of water in graphene nano-channels. Microfluid Nanofluid, 2018, 22, P. 7.

[4] Erfle P., Riewe J., Bunjes H., Dietzel A. Optically monitored segmented flow for controlled ultra-fast mixing and nanoparticle precipitation. Microfluid Nanofluid, 2017, 21, P. 179.

[5] Ghasemi A., Amiri H., Zare H., Masroor M., Hasanzadeh A., Beyzavi A., Aref A.R., Karimi M., Hamblin M.R. Carbon nanotubes in microfluidic lab-on-a-chip technology: current trends and future perspectives. Microfluid Nanofluid, 2017,21, P. 151.

[6] Kosheleva O.K., Lai T.C, Chen N.G., Hsiao V., Chen Ch.H. Selective killing of cancer cells by nanoparticle.assisted ultrasound. J. Nanobiotechnol., 2016, 14, P. 46.

[7] Okamoto R., Kanemori Y., Komura Sh., Fournier J.-B. Relaxation dynamics of two-component fluid bilayer membranes. Eur. Phys. J. E, 2016, 39, P. 52.

[8] Hackborn W.W. Asymmetric Stokes flow between parallel planes due to a rotlet. J. Fluid Mech., 1990, 218, P. $631-546$.

[9] Pozrikidis C. Computation of periodic Green's functions of Stokes flow. J. Engineering Math., 1996, 30, P. 79-96.

[10] Gugel Yu.V., Popov I.Yu., Popova S.L. Hydrotron: creep and slip. Fluid. Dyn. Res., 1996, 18(4), P. $199-210$.

[11] Popov I.Y., Blinova I.V., Kyz’yurova K.N. Stokes flow driven by a Stokeslet in a cone. Acta Mechanica, 2014, 225, P. 3115-3121.

[12] Landau L. D., Lifshitz E. M. Fluid Mechanics, 2nd ed., Vol. 6. Pergamon Press, Oxford.: 1993. 532 p.

[13] Ribe, N. M. Bending and stretching of thin viscous sheets. Journal of Fluid Mechanics, 2001, 433, P. 135-160.

[14] Ribe, N. M.A general theory for the dynamics of thin viscous sheets. Journal of Fluid Mechanics, 2002, 457, P. $255-283$.

[15] Pfingstag, G., Audoly, B., Boudaoud, A. Linear and nonlinear stability of floating viscous sheets. Journal of Fluid Mechanics, 2011, 683, P. $112-148$.

[16] Timoshenko, S., Woinowsky-Krieger, S. Theory of plates and shells. McGraw-Hill, New York.: 1959, P. 595 p. 